\title{
Multihomogeneous Polynomial Decomposition using Moment Matrices
}

\author{
Alessandra Bernardi* Jérôme Brachat* \\ *GALAAD, INRIA Méditerranée \\ BP 93, 06902 Sophia-Antipolis, France \\ [FirstName.LastName]@inria.fr
}

\author{
Pierre Comon ${ }^{+} \quad$ Bernard Mourrain* \\ +Laboratoire I3S, CNRS and Univ. of Nice, \\ Sophia-Antipolis, France \\ pcomon@i3s.unice.fr
}

\begin{abstract}
In the paper, we address the important problem of tensor decomposition which can be seen as a generalisation of Singular Value Decomposition for matrices. We consider general multilinear and multihomogeneous tensors. We show how to reduce the problem to a truncated moment matrix problem and we give a new criterion for flat extension of Quasi-Hankel matrices. We connect this criterion to the commutation characterisation of border bases. A new algorithm is described: it applies for general multihomogeneous tensors, extending the approach of J.J. Sylvester on binary forms. An example illustrates the algebraic operations involved in this approach and how the decomposition can be recovered from eigenvector computation.
\end{abstract}

\section{Categories and Subject Descriptors}

G.1.5 [Mathematics of Computing]: Tensor decomposition; I.1.2 [Computing Methodologies]: Symbolic and Algebraic Manipulation-Algebraic algorithms

\section{General Terms}

Algorithms, Theory

\section{Keywords}

Moment matrix, multihomogeneous polynomial decomposition, tensor decomposition.

\section{INTRODUCTION}

Tensors are objects which appear in many context and applications. The most famous type of tensors corresponds to matrices which are tensors of order two. However in many problems, higher order tensors are naturally used to collect informations which depend on more than two variables. Typically, these data could be observations of some experimentation or of a physical phenomena that depends on several parameters. These observations are stored in a structure called a tensor, according to the dimensional parameters (or modes) of the problem.

Permission to make digital or hard copies of all or part of this work for personal or classroom use is granted without fee provided that copies are not made or distributed for profit or commercial advantage and that copies bear this notice and the full citation on the first page. To copy otherwise, to republish, to post on servers or to redistribute to lists, requires prior specific permission and/or a fee.

ISSAC'11, June 8-11, 2011, San Jose, California, USA

Copyright 2011 ACM 978-1-4503-0675-1/11/06 ...\$10.00.
The tensor decomposition problem consists to decompose the tensor (e.g. the set of observations) into a minimal sum of indecomposable tensors (i.e. tensors of rank 1). Such a decomposition, which is independent of the coordinate system, allows to extract geometric or invariant properties associated to the observations. For this reason, the tensor decomposition problem has a large impact in many applications. The first well known case for matrices is related to Singular Value Decomposition with applications e.g. to Principal Component Analysis. Its extension to higher order tensors appears in Electrical Engineering [44], in Signal processing [17], [12], in Antenna Array Processing [21] [11] or Telecommunications [46], [10], [41], [24], [20], in Chemometrics [6] or Psychometrics [29], in Data Analysis [14], [9], [22], [28], [42], but also in more theoretical domains such as Arithmetic complexity [30] [4], [43], [31]. Further numerous applications of tensor decompositions may be found in [12], [42].

From a mathematical point of view, the tensors that we will consider are elements of $\mathcal{T}:=S^{\delta_{1}}\left(E_{1}\right) \otimes \cdots \otimes S^{\delta_{k}}\left(E_{k}\right)$ where $\delta_{i} \in \mathbb{N}, E_{i}$ are vector spaces of dimension $n_{i}+1$ over a field $\mathbb{K}$ (which is of characteristic 0 and algebraically closed), and $S^{\delta_{i}}\left(E_{i}\right)$ is the $\delta_{i}^{\text {th }}$ symmetric power of $E_{i}$. The set of tensors of rank 1 form a projective variety which is called the Veronese variety when $k=1$ or the Segre variety when $\delta_{i}=1, i=1, \ldots, k$. We will call it hereafter the SegreVeronese variety of $\mathbb{P}(\mathcal{T})$ and denote it $\Xi(\mathcal{T})$. The set of tensors which are the linear combinations of $r$ elements of the Segre-Veronese variety are those which admits a decomposition with at most $r$ terms of rank 1 (ie. in $\Xi(\mathcal{T})$ ). The closure of this set is called the $r$-secant variety and denoted $\Xi_{r}(\mathcal{T})$. More precise definitions of these varieties will be given in Sec. 2.3.

The first method to compute such a decomposition besides the case of matrices or quadratic forms which may go back to the Babylonians, is due to Sylvester for binary forms [45]. Using apolarity, kernels of catalecticant matrices are computed degree by degree until a polynomial with simple roots is found. See also [13], [27]. An extension of this approach for symmetric tensors has been analyzed in [27], and yields a decomposition method in some cases (see [27][p. 187]). Some decomposition methods are also available for specific degrees and dimensions, e.g. using invariant theory [16]. In [3], there is a simplified version of the Sylvester algorithm that uses the mathematical interpretation of the problem in terms of secant varieties of rational normal curves. The same approach is used in [3] to give algorithms for the de- 
compositions of symmetric tensors belonging to $\Xi_{2}\left(S^{d}(E)\right)$ and to $\Xi_{3}\left(S^{d}(E)\right)$. In [1] a complete rank stratification of $\Xi_{4}\left(S^{d}(E)\right)$ is given.

In [5], Sylvester's approach is revisited from an affine point of view and a general decomposition method based on a flat extension criteria is described. In the current paper, we extend this method to more general tensor spaces including classical multilinear tensors and multihomogeneous tensors. We give a new and more flexible criterion for the existence of a decomposition of a given rank, which extend the result in [35] and the characterisation used in [5]. This criterion is a rank condition of an associated Hankel operator. It is used in an algorithm which checks degree by degree if the roots deduced from the kernel of the Hankel operator are simple.

In Sec. 2, we recall the notations, the geometric point related to secants of Segre and Veronese varieties, and the algebraic point of view based on moment matrices. In Sec. 3 , we describe the algorithm and the criterion used to solve the truncated moment problem. In Sec. 4, an example of tensor decompositions from Antenna processing illustrates the approach.

\section{DUALITY, MOMENT MATRICES AND TENSOR DECOMPOSITION}

\subsection{Notation and preliminaries}

Let $\mathbb{K}$ be an algebraically closed field (e.g. $\mathbb{K}=\mathbb{C}$ the field of complex numbers). We assume that $\mathbb{K}$ is of characteristic 0 . For a vector space $E$, its associated projective space is denoted $\mathbb{P}(E)$. For $\mathbf{v} \in E-\{0\}$ its class in $\mathbb{P}(E)$ is denoted $\overline{\mathbf{v}}$. Let $\mathbb{P}^{n}$ be the projective space of $E:=\mathbb{K}^{n+1}$. For a subset $F=\left\{f_{1}, \ldots, f_{m}\right\}$ of a vector-space (resp. ring) $R$, we denote by $\langle F\rangle$ (resp. $(F))$ the vector space (resp. ideal) generated by $F$ in $R$.

We consider hereafter the symmetric $\delta$-th power $S^{\delta}(E)$ where $E$ is a vector space of basis $x_{0}, \ldots, x_{n}$. An element of $S^{\delta}(E)$ is a homogeneous polynomial of degree $\delta \in \mathbb{N}$ in the variables $\mathbf{x}=\left(x_{0}, \ldots, x_{n}\right)$. For $\mathbf{x}_{1}=\left(x_{0,1}, \ldots, x_{n_{1}, 1}\right)$ $, \ldots, \mathbf{x}_{k}=\left(x_{0, k}, \ldots, x_{n_{k}, k}\right), S^{\delta_{1}}\left(E_{1}\right) \otimes \cdots \otimes S^{\delta_{k}}\left(E_{k}\right)$ (with $\left.E_{i}=\left\langle x_{0, i}, \ldots, x_{n_{i}, i}\right\rangle\right)$ is the vector space of multihomogeneous polynomials of degree $\delta_{i}$ in the variables $\mathbf{x}_{i}$.

Hereafter, we will consider the dehomogeneization of elements in $S^{\delta_{1}}\left(E_{1}\right) \otimes \cdots \otimes S^{\delta_{k}}\left(E_{k}\right)$, obtained by setting $x_{0, i}=$ 1 for $i=1, \ldots, k$. We denote by $R_{\delta_{1}, \ldots, \delta_{k}}$ this space, where $R=\mathbb{K}\left[\underline{\mathbf{x}}_{1}, \ldots, \underline{\mathbf{x}}_{k}\right]$ is the space of polynomials in the variables $\underline{\mathbf{x}}_{1}=\left(x_{1,1}, \ldots, x_{n_{1}, 1}\right), \ldots, \underline{\mathbf{x}}_{k}=\left(x_{1, k}, \ldots, x_{n_{k}, k}\right)$.

For $\alpha_{i}=\left(\alpha_{1, i}, \ldots, \alpha_{n_{i}, i}\right) \in \mathbb{N}^{n_{i}}(i=1, \ldots, k)$, let $\underline{\mathbf{x}}_{i}^{\alpha_{i}}=$ $\prod_{j=1}^{n_{i}} x_{j, i}^{\alpha_{j, i}},\left|\alpha_{\mathbf{i}}\right|=\sum_{j=1}^{n_{i}} \alpha_{j, i}$, and $\underline{\mathbf{x}}^{\alpha}=\prod_{j=1}^{n_{i}} \mathbf{x}_{i}^{\alpha_{i}}$.

An element $f$ of $R_{\delta}=R_{\delta_{1}, \ldots, \delta_{k}}$ is represented as

$$
f=\sum_{\alpha=\left(\alpha_{1}, \ldots, \alpha_{k}\right) ;\left|\alpha_{i}\right| \leq \delta_{i}} f_{\alpha} \underline{\mathbf{x}}_{1}^{\alpha_{1}} \cdots \underline{\mathbf{x}}_{k}^{\alpha_{k}} .
$$

The dimension of $R_{\delta}:=R_{\delta_{1}, \ldots, \delta_{k}}$ is $n_{\delta_{1}, \ldots, \delta_{k} ; n_{1}, \ldots, n_{k}}=$ $\prod_{i=1}^{k}\left(\begin{array}{c}n_{i}+\delta_{i} \\ \delta_{i}\end{array}\right)$. For $\delta \in \mathbb{N}, \alpha \in \mathbb{N}^{n}$ with $|\alpha| \leq \delta$, let $\left(\begin{array}{l}\delta \\ \alpha\end{array}\right)=$ $\frac{\delta !}{\alpha_{1} ! \cdots \alpha_{n} !(\delta-|\alpha|) !}$. We define the apolar inner product on $R_{\delta_{1}, \ldots, \delta_{k}}$ by $\langle f \mid g\rangle=\sum_{\left|\alpha_{i}\right| \leq \delta_{i}} f_{\alpha} g_{\alpha}\left(\begin{array}{c}\delta_{1} \\ \alpha_{1}\end{array}\right)^{-1} \cdots\left(\begin{array}{c}\delta_{k} \\ \alpha_{k}\end{array}\right)^{-1}$.

The dual space of a $\mathbb{K}$-vector space $E$ is denoted $E^{*}=$ $\operatorname{Hom}_{\mathbb{K}}(E, \mathbb{K})$. It is the set of $\mathbb{K}$-linear forms from $E$ to $\mathbb{K}$. A basis of the dual space $R_{\delta}^{*}$, is given by the set of linear forms that compute the coefficients of a polynomial in the monomial basis $\left(\underline{\mathbf{x}}^{\alpha}\right)_{\alpha \in \mathbb{N}^{n} 1 \times \cdots \times \mathbb{N}^{n} k ;\left|\alpha_{i}\right| \leq \delta_{i}}$. We denote it

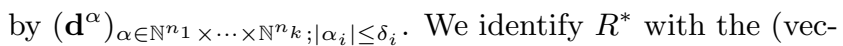
tor) space of formal power series $\mathbb{K}[[\mathbf{d}]]=\mathbb{K}\left[\left[\mathbf{d}_{1}, \ldots, \mathbf{d}_{k}\right]\right]=$ $\mathbb{K}\left[\left[d_{1,1}, \ldots, d_{n_{1}, 1}, \ldots, d_{1, k}, \ldots, d_{n_{k}, k}\right]\right]$. Any element $\Lambda \in R^{*}$ can be decomposed as

$$
\Lambda=\sum_{\alpha \in \mathbb{N}^{n} 1 \times \cdots \times \mathbb{N}^{n} k} \Lambda\left(\mathbf{x}^{\alpha}\right) \mathbf{d}^{\alpha} .
$$

Typical elements of $R^{*}$ are the linear forms that correspond to the evaluation at a point $\zeta=\left(\zeta_{1}, \ldots, \zeta_{k}\right) \in$ $\mathbb{K}^{n_{1}} \times \cdots \times \mathbb{K}^{n_{k}}$ :

$$
\begin{aligned}
\mathbf{1}_{\zeta} \quad: \quad & R \rightarrow \mathbb{K} \\
& p \mapsto p(\zeta)
\end{aligned}
$$

The decomposition of $\mathbf{1}_{\zeta}$ in the basis $\left\{\mathbf{d}^{\alpha}\right\}_{\alpha \in \mathbb{N}^{n_{1}} \times \cdots \times \mathbb{N}^{n} k}$ is

$$
\mathbf{1}_{\zeta}=\sum_{\alpha \in \mathbb{N}^{n} 1 \times \cdots \times \mathbb{N}^{n_{k}}} \zeta^{\alpha} \mathbf{d}^{\alpha}=\sum_{\alpha \in \mathbb{N}^{n}} \prod_{1 \times \cdots \times \mathbb{N}^{n} k} \prod_{i=1}^{k} \zeta_{i}^{\alpha_{i}} \mathbf{d}_{i}^{\alpha_{i}} .
$$

We recall that the dual space $R^{*}$ has a natural structure of $R$-module [23] which is defined as follows: for all $p \in R$, and for all $\Lambda \in R^{*}$ consider the linear operator

$$
\begin{aligned}
p \star \Lambda: & R \rightarrow \mathbb{K} \\
& q \mapsto \Lambda(p q) .
\end{aligned}
$$

In particular, we have $x_{i, j} \star \mathbf{d}_{1}^{\alpha_{1}} \cdots \mathbf{d}_{j}^{\alpha_{j}} \cdots \mathbf{d}_{k}^{\alpha_{k}}=$

$\mathbf{d}_{1}^{\alpha_{1}} \cdots \mathbf{d}_{j-1}^{\alpha_{j-1}} d_{1, j}^{\alpha_{1, j}} \cdots d_{i-1, j}^{\alpha_{i-1, j}} d_{i, j}^{\alpha_{i, j}-1} d_{i+1, j}^{\alpha_{i+1, j}} \cdots d_{n_{j}, j}^{\alpha_{n_{j}, j}} \mathbf{d}_{j+1}^{\alpha_{j+1}} \cdots \mathbf{d}_{k}^{\alpha_{k}}$ if $\alpha_{i, j}>0$ and 0 otherwise.

\subsection{Tensor decomposition}

In this section, we present different formulations of the tensor decomposition problem, that we consider in this paper.

We will consider hereafter a partially symmetric tensor $T$ which is an element of $S^{\delta_{1}}\left(E_{1}\right) \otimes \cdots \otimes S^{\delta_{k}}\left(E_{k}\right)$ where $E_{i}=\left\langle x_{0, i}, \ldots, x_{n_{i}, i}\right\rangle$. It can be represented by a partially symmetric array of coefficients

$$
[T]=\left(T_{\alpha_{1}, \ldots, \alpha_{k}}\right)_{\alpha_{i} \in \mathbb{N}^{n_{i}+1} ;\left|\alpha_{i}\right|=\delta_{i}} .
$$

For $\alpha_{i} \in \mathbb{N}^{n_{i}}$ with $\left|\alpha_{i}\right| \leq \delta_{i}$, we denote $\bar{\alpha}_{i}=\left(\delta_{i}-\left|\alpha_{i}\right|, \alpha_{1, i}, \ldots\right.$, $\left.\alpha_{n_{i}, i}\right)$ and, with an abuse of notation, we identify $T_{\alpha_{1}, \ldots, \alpha_{k}}:=$ $T_{\overline{\alpha_{1}}, \ldots, \overline{\alpha_{k}}}$.

Such a tensor is naturally associated to a (multihomogeneous) polynomial in the variables $\mathbf{x}_{1}=\left(x_{0,1}, \ldots, x_{n_{1}, 1}\right)$, $\ldots, \mathbf{x}_{k}=\left(x_{0, k}, \ldots, x_{n_{k}, k}\right)$

$$
T(\mathbf{x})=\sum_{\substack{\alpha=\left(\alpha_{1}, \ldots, \alpha_{k}\right) \in \mathbb{N}^{n_{1}}\left|\alpha_{i}\right| \leq \delta_{i} \\\left(x \times \mathbb{N}^{n} k\right.}} T_{\alpha} \mathbf{x}_{1}^{\bar{\alpha}_{1}} \cdots \mathbf{x}_{k}^{\bar{\alpha}_{k}} .
$$

or to an element $\underline{T}(\underline{\mathbf{x}}) \in R_{\delta_{1}, \ldots, \delta_{k}}$ obtained by substituting $x_{0, i}$ by 1 in $T(\mathbf{x})$ (for $\left.i=1, \ldots, k\right)$ :

$$
\underline{T}(\underline{\mathbf{x}})=\sum_{\substack{\alpha \in \mathbb{N}^{n} \\\left|\alpha_{i}\right| \leq \delta_{i}}} T_{\alpha} \underline{\mathbf{x}}_{1}^{\alpha_{1}} \cdots \underline{\mathbf{x}}_{k}^{\alpha_{k}} .
$$

An element of $R^{*}=\mathbb{K}[[\mathbf{d}]]$ can also be associated naturally to $T$ :

$$
T^{*}(\mathbf{d})=\sum_{\substack{\alpha \in \mathbb{N}^{n} 1 \times \cdots \times \mathbb{N}^{n} k \\
\left|\alpha_{i}\right| \leq \delta_{i}}}\left(\begin{array}{c}
\delta_{1} \\
\alpha_{1}
\end{array}\right)^{-1} \cdots\left(\begin{array}{c}
\delta_{k} \\
\alpha_{k}
\end{array}\right)^{-1} T_{\alpha} \mathbf{d}_{1}^{\alpha_{1}} \cdots \mathbf{d}_{k}^{\alpha_{k}} .
$$

so that for all $T^{\prime} \in R_{\delta_{1}, \ldots, \delta_{k}}$,

$$
\left\langle T(\underline{\mathbf{x}}) \mid T^{\prime}(\underline{\mathbf{x}})\right\rangle=T^{*}(\mathbf{d})\left(T^{\prime}(\underline{\mathbf{x}})\right)
$$


The problem of decomposition of the tensor $T$ can be stated as follows:

Tensor decomposition problem. Given $T(\mathbf{x}) \in S^{\delta_{1}}\left(E_{1}\right)$ $\otimes \cdots \otimes S^{\delta_{k}}\left(E_{k}\right)$, find a decomposition of $T(\mathbf{x})$ as a sum of products of powers of linear forms in $\mathbf{x}_{j}$ :

$$
T(\mathbf{x})=\sum_{i=1}^{r} \gamma_{i} \mathbf{l}_{1, i}\left(\mathbf{x}_{1}\right)^{\delta_{1}} \cdots \mathbf{l}_{k, i}\left(\mathbf{x}_{k}\right)^{\delta_{k}}
$$

where $\gamma_{i} \neq 0, \mathbf{l}_{j, i}\left(\mathbf{x}_{j}\right)=l_{0, j, i} x_{0, j}+l_{1, j, i} x_{1, j}+\cdots+l_{n_{j}, j, i} x_{j, n_{j}}$ and $r$ is the smallest possible integer for such a decomposition.

DeFINITION 2.1. The minimal number of terms $r$ in a decomposition of the form (2) is called the rank of $T$.

We say that $T(\mathbf{x})$ has an affine decomposition if there exists a minimal decomposition of $T(\mathbf{x})$ of the form (2) where $r$ is the rank of $T$ and such that $l_{0, j, i} \neq 0$ for $i=1, \ldots, r$. Notice that by a generic change of coordinates in $E_{i}$, we may assume that all $l_{0, j, i} \neq 0$ and thus that $T$ has an affine decomposition. Suppose that $T(\mathbf{x})$ has an affine decomposition. Then by scaling $\mathbf{l}_{j, i}\left(\mathbf{x}_{j}\right)$ and multiplying $\gamma_{i}$ by the inverse of the $\delta_{j}^{t h}$ power of this scaling factor, we may assume that $l_{0, j, i}=1$. Thus, the polynomial

$\underline{T}(\underline{\mathbf{x}})=\sum_{i=1}^{r} \gamma_{i} \sum_{\left|\alpha_{i}\right| \leq \delta_{i}}\left(\begin{array}{c}\delta_{1} \\ \alpha_{1}\end{array}\right) \cdots\left(\begin{array}{c}\delta_{k} \\ \alpha_{k}\end{array}\right) \zeta_{1, i}^{\alpha_{1}} \cdots \zeta_{k, i}^{\alpha_{k}} \underline{\mathbf{x}}_{1}^{\alpha_{1}} \cdots \underline{\mathbf{x}}_{k}^{\alpha_{k}}$

with $T_{\alpha_{1}, \ldots, \alpha_{k}}=\sum_{i=1}^{r} \gamma_{i} \sum_{\left|\alpha_{i}\right| \leq \delta_{i}}\left(\begin{array}{c}\delta_{1} \\ \alpha_{1}\end{array}\right) \cdots\left(\begin{array}{c}\delta_{k} \\ \alpha_{k}\end{array}\right) \zeta_{1, i}^{\alpha_{1}} \cdots \zeta_{k, i}^{\alpha_{k}}$. Equivalently, we have

$$
T^{*}(\mathbf{d})=\sum_{i=1}^{r} \gamma_{i} \sum_{\left|\alpha_{i}\right| \leq \delta_{i}} \zeta_{1, i}^{\alpha_{1}} \cdots \zeta_{k, i}^{\alpha_{k}} \mathbf{d}_{1}^{\alpha_{1}} \cdots \mathbf{d}_{k}^{\alpha_{k}}
$$

so that $T^{*}(\mathbf{d})$ coincides on $R_{\delta_{1}, \ldots, \delta_{k}}$ with the linear form

$$
\sum_{i=1}^{r} \gamma_{i} \mathbf{1}_{\zeta_{1, i}, \ldots, \zeta_{k, i}}=\sum_{i=1}^{r} \gamma_{i} \mathbf{1}_{\zeta_{i}}
$$

with $\zeta_{i}:=\left(\zeta_{1, i}, \ldots, \zeta_{k, i}\right) \in \mathbb{K}^{n_{1}} \times \cdots \mathbb{K}^{n_{k}}$.

The problem of decomposition of $T$ can then be restated as follows:

Interpolation problem. Given $T^{*} \in R_{\delta_{1} \ldots . \delta_{k}}^{*}$ which admits an affine decomposition, find the minimal number of non-zero vectors $\zeta_{1}, \ldots, \zeta_{r} \in \mathbb{K}^{n_{1}} \times \cdots \times \mathbb{K}^{n_{k}}$ and non-zero scalars $\gamma_{1}, \ldots, \gamma_{r} \in \mathbb{K}-\{0\}$ such that

$$
T^{*}=\sum_{i=1}^{r} \gamma_{i} \mathbf{1}_{\zeta_{i}}
$$

on $R_{\delta_{1}, \ldots, \delta_{k}}$.

If such a decomposition exists, we say that $\Lambda=\sum_{i=1}^{r} \gamma_{i} \mathbf{1}_{\zeta_{i}} \in$ $R^{*}$ extends $T^{*} \in R_{\delta_{1}, \ldots, \delta_{k}}^{*}$

\subsection{Indecomposable tensors}

In this section, we analyze the set of indecomposable tensor (or tensors of rank 1). They naturally form projective varieties, which we are going to describe using the language of projective geometry.

We begin by defining two auxiliary but very classical varieties, namely Segre variety and Veronese variety.

Definition 2.2. The image of the following map

$$
\begin{aligned}
s_{k}: \mathbb{P}\left(E_{1}\right) \times \cdots \times \mathbb{P}\left(E_{k}\right) & \rightarrow \mathbb{P}\left(E_{1} \otimes \cdots \otimes E_{k}\right) \\
\left(\overline{\mathbf{v}_{1}}, \ldots, \overline{\mathbf{v}_{k}}\right) & \mapsto \overline{\mathbf{v}_{1} \otimes \cdots \otimes \mathbf{v}_{k}}
\end{aligned}
$$

is the so called Segre variety of $k$ factors. We denote it by $\Xi\left(E_{1} \otimes \cdots \otimes E_{k}\right)$.

From Definition 2.1 of the rank of a tensor and from the Interpolation Problem point of view (3) we see that a Segre variety parametrizes projective classes of rank 1 tensors $T=$ $\mathbf{v}_{\mathbf{1}} \otimes \cdots \otimes \mathbf{v}_{\mathbf{k}} \in E_{1} \otimes \cdots \otimes E_{k}$ for certain $\mathbf{v}_{\mathbf{i}} \in E_{i}, i=1, \ldots, k$.

DeFinition 2.3. Let $\left(J_{1}, J_{2}\right)$ be a partition of the set $\{1, \ldots, k\}$. If $J_{1}=\left\{h_{1}, \ldots, h_{s}\right\}$ and $J_{2}=\{1, \ldots, k\} \backslash J_{1}=\left\{h_{1}^{\prime}, \ldots, h_{k-s}^{\prime}\right\}$, the $\left(J_{1}, J_{2}\right)$-Flattening of $E_{1} \otimes \cdots \otimes E_{k}$ is the following:

$$
E_{J_{1}} \otimes E_{J_{2}}=\left(E_{h_{1}} \otimes \cdots \otimes E_{h_{s}}\right) \otimes\left(E_{h_{1}^{\prime}} \otimes \cdots \otimes E_{h_{k-s}^{\prime}}\right) .
$$

Let $E_{J_{1}} \otimes E_{J_{2}}$ be any flattening of $E_{1} \otimes \cdots \otimes E_{k}$ as in Definition 2.3 and let $f_{J_{1}, J_{2}}: \mathbb{P}\left(E_{1} \otimes \cdots \otimes E_{k}\right) \rightarrow \mathbb{P}\left(E_{J_{1}} \otimes E_{J_{2}}\right)$ be the obvious isomorphism. Let $[T]$ be an array associated to a tensor $T \in E_{1} \otimes \cdots \otimes E_{k}$; let $\overline{T^{\prime}}=f_{J_{1}, J_{2}}(\overline{\mathbf{T}}) \in \mathbb{P}\left(E_{J_{1}} \otimes E_{J_{2}}\right)$ and let $\left[A_{J_{1}, J_{2}}\right]$ be the matrix associated to $T^{\prime}$. Then the $d$-minors of the matrix $\left[A_{J_{1}, J_{2}}\right]$ are said to be $d$-minors of $[T]$.

An array $[A]=\left(x_{i_{1}, \ldots, i_{k}}\right)_{0 \leq i_{j} \leq n_{j}, j=1, \ldots, k}$ is said to be a generic array of indeterminates of $R=\mathbb{K}\left[\underline{\mathbf{x}}_{1}, \ldots, \underline{\mathbf{x}}_{k}\right]$ if the entries of $[A]$ are the independent variables of $R$.

It is a classical result due to R. Grone (see [25]) that a set of equations for a Segre variety is given by all the 2-minors of a generic array. In [26] it is proved that, if $[A]$ is a generic array in $R$ of size $\left(n_{1}+1\right) \times \cdots \times\left(n_{k}+1\right)$ and $I_{d}([A])$ is the ideal generated by the $d$-minors of $[A]$, then $I_{2}([A])$ is a prime ideal, therefore:

$$
I\left(\Xi\left(E_{1} \otimes \cdots \otimes E_{k}\right)\right)=I_{2}([A]) .
$$

We introduce now the Veronese variety. Classically it is defined to be the $d$-tuple embedding of $\mathbb{P}^{n}$ into $\mathbb{P}^{\left(\begin{array}{c}n+d \\ d\end{array}\right)-1}$ via the linear system associated to the sheaf $\mathcal{O}(d)$ with $d>0$. We give here an equivalent definition.

Let $E$ be an $n+1$ dimensional vector space. With the notation $S^{d}(E)$ we mean the vector subspace of $E^{\otimes d}$ of symmetric tensors.

Definition 2.4. The image of the following map

$$
\begin{aligned}
\nu_{d}: \mathbb{P}(E) & \rightarrow \mathbb{P}\left(S^{d}(E)\right) \\
\overline{\mathbf{v}} & \mapsto \overline{\mathbf{v}} \otimes \mathbf{d}
\end{aligned}
$$

is the so called Veronese variety. We indicate it with $\Xi\left(S^{d}(E)\right)$.

With this definition it is easy to see that the Veronese variety parametrizes symmetric rank 1 tensors.

Observe that if we take the vector space $E$ to be a vector space of linear forms $\left\langle x_{0}, \ldots, x_{n}\right\rangle$ then the image of the map $\nu_{d}$ above parametrizes homogeneous polynomials that can be written as $d$-th powers of linear forms.

The Veronese variety $\Xi\left(S^{d}(E)\right) \subset \mathbb{P}\left(S^{d}(E)\right)$ can be also viewed as $\Xi\left(S^{d}(E)\right)=\Xi\left(E^{\otimes d}\right) \cap \mathbb{P}\left(S^{d}(E)\right)$.

Let $[A]=\left(x_{i_{1}, \ldots, i_{d}}\right)_{0 \leq i_{j} \leq n, j=1, \ldots, d}$ be a generic symmetric array. It is a known result that:

$$
I\left(\Xi\left(S^{d}(E)\right)\right)=I_{2}([A]) .
$$

See [47] for the set theoretical point of view. In [39] the author proved that $I\left(\Xi\left(S^{d}(E)\right)\right)$ is generated by the 2-minors of a particular catalecticant matrix (for a definition of "Catalecticant matrices" see e.g. either [39] or [?]). A. Parolin, in his $\mathrm{PhD}$ thesis ([38]), proved that the ideal generated by the 2 -minors of that catalecticant matrix is actually $I_{2}([A])$. 
We are now ready to describe the geometric object that parametrizes partially symmetric tensors $T \in S^{\delta_{1}}\left(E_{1}\right) \otimes \cdots \otimes$ $S^{\delta_{k}}\left(E_{k}\right)$. Let us start with the rank 1 partially symmetric tensors.

Definition 2.5. Let $E_{1}, \ldots, E_{k}$ be vector spaces of dimensions $n_{1}+1, \ldots, n_{k}+1$ respectively. The Segre-Veronese variety $\Xi\left(S^{\delta_{1}}\left(E_{1}\right) \otimes \cdots \otimes S^{\delta_{k}}\left(E_{k}\right)\right)$ is the embedding of $\mathbb{P}\left(E_{1}\right) \otimes$ $\cdots \otimes \mathbb{P}\left(E_{k}\right)$ into $\mathbb{P}^{N-1} \simeq \mathbb{P}\left(S^{\delta_{1}}\left(E_{1}\right) \otimes \cdots \otimes S^{\delta_{k}}\left(E_{k}\right)\right)$, where $N=\left(\prod_{i=1}^{k}\left(\begin{array}{c}n_{i}+\delta_{i} \\ d_{i}\end{array}\right)\right)$, given by sections of the sheaf $\mathcal{O}\left(\delta_{1}, \ldots, \delta_{k}\right)$. I.e. $\Xi\left(S^{\delta_{1}}\left(E_{1}\right) \otimes \cdots \otimes S^{\delta_{k}}\left(E_{k}\right)\right)$ is the image of the composition of the following two maps:

$\left.\mathbb{P}\left(E_{1}\right) \times \cdots \times \mathbb{P}\left(E_{k}\right) \stackrel{\nu_{\delta_{1}} \times \cdots \times \nu_{\delta_{k}}}{\longrightarrow} \mathbb{P}^{\left({ }_{1}+\delta_{\delta_{1}}\right.}\right)-1 \times \cdots \times \mathbb{P}^{\left({ }^{n_{k}+\delta_{k}} \delta_{\delta_{k}}\right)-1}$ and $\mathbb{P}^{\left(\begin{array}{c}n_{1}+\delta_{1} \\ \delta_{1}\end{array}\right)-1} \times \cdots \times \mathbb{P}^{\left(\begin{array}{c}n_{k}+\delta_{k} \\ \delta_{t}\end{array}\right)-1} \stackrel{s}{\longrightarrow} \mathbb{P}^{N-1}$, where each $\nu_{\delta_{i}}$ is a Veronese embedding of $\mathbb{P}\left(E_{i}\right)$ as in Definition 2.4 then $\operatorname{Im}\left(\nu_{\delta_{1}} \times \cdots \times \nu_{\delta_{k}}\right)=\Xi\left(S^{\delta_{1}}\left(E_{1}\right)\right) \times \cdots \times \Xi\left(S^{\delta_{k}}\left(E_{k}\right)\right)$ and $\operatorname{Im}(s)$ is the Segre variety of $k$ factors. Therefore the SegreVeronese variety is the Segre re-embedding of the product of $k$ Veronese varieties.

If $\left(\delta_{1}, \ldots, \delta_{k}\right)=(1, \ldots, 1)$ then the corresponding SegreVeronese variety is nothing else than the classical Segre variety of $\mathbb{P}\left(E_{1} \otimes \cdots \otimes E_{k}\right)$.

If $k=1$ then the corresponding Segre-Veronese variety is nothing else than the classical Veronese variety of $\mathbb{P}\left(S^{\delta_{1}}\left(E_{1}\right)\right)$.

Observe that $\Xi\left(S^{\delta_{1}}\left(E_{1}\right) \otimes \cdots \otimes S^{\delta_{k}}\left(E_{k}\right)\right)$ can be viewed as the intersection with the Segre variety $\Xi\left(E_{1}^{\otimes \delta_{1}} \otimes \cdots \otimes\right.$ $E_{k}^{\otimes \delta_{k}}$ ) that parametrizes rank one tensors and the projective subspace $\mathbb{P}\left(S^{\delta_{1}}\left(E_{1}\right) \otimes \cdots \otimes S^{\delta_{k}}\left(E_{k}\right)\right) \subset \mathbb{P}\left(E_{1}^{\otimes \delta_{1}} \otimes \cdots \otimes E_{k}^{\otimes \delta_{k}}\right)$ that parametrizes partially symmetric tensors: $\Xi\left(S^{\delta_{1}}\left(E_{1}\right) \otimes\right.$ $\left.\cdots \otimes S^{\delta_{k}}\left(E_{k}\right)\right)=\Xi\left(E_{1}^{\otimes \delta_{1}} \otimes \cdots \otimes E_{k}^{\otimes \delta_{k}}\right) \cap \mathbb{P}\left(S^{\delta_{1}}\left(E_{1}\right) \otimes \cdots \otimes\right.$ $\left.S^{\delta_{k}}\left(E_{k}\right)\right)$.

In [2] it is proved that if $[A]$ is a generic array of indeterminates associated to the multihomogeneous polynomial ring $S^{\delta_{1}}\left(E_{1}\right) \otimes \cdots \otimes S^{\delta_{k}}\left(E_{k}\right)$ (i.e. it is a generic partially symmetric array), the ideal of the Segre-Veronese variety $\Xi\left(S^{\delta_{1}}\left(E_{1}\right) \otimes \cdots \otimes S^{\delta_{k}}\left(E_{k}\right)\right)$ is

$$
I\left(\Xi\left(S^{\delta_{1}}\left(E_{1}\right) \otimes \cdots \otimes S^{\delta_{k}}\left(E_{k}\right)\right)\right)=I_{2}([A])
$$

with $\delta_{i}>0$ for $i=1, \ldots, k$.

Now if we consider the vector spaces $E_{i}$ that are vector spaces of linear forms $E_{i} \simeq S^{1}\left(E_{i}\right)$ for $i=1, \ldots, k$, we get that the Segre-Veronese variety $\Xi\left(S^{\delta_{1}}\left(E_{1}\right) \otimes \cdots \otimes S^{\delta_{k}}\left(E_{k}\right)\right)$ parametrizes multihomogenoeus polynomials $F \in S^{\delta_{1}}\left(E_{1}\right) \otimes$ $\cdots \otimes S^{\delta_{k}}\left(E_{k}\right)$ of the type $F=\mathbf{l}_{1}^{\delta_{1}} \cdots \mathbf{l}_{k}^{\delta_{k}}$ where $\mathbf{l}_{i}$ are linear forms in $S^{1}\left(E_{i}\right)$ for $i=1, \ldots, k$.

From this observation we understand that the Tensor decomposition problem of finding a minimal decomposition of type (2) for an element $T \in S^{\delta_{1}}\left(E_{1}\right) \otimes \cdots \otimes S^{\delta_{k}}\left(E_{k}\right)$ is equivalent to finding the minimum number of elements belonging to the Segre-Veronese variety $\Xi\left(S^{\delta_{1}}\left(E_{1}\right) \otimes \cdots \otimes S^{\delta_{k}}\left(E_{k}\right)\right)$ whose span contains $\bar{T} \in \mathbb{P}\left(S^{\delta_{1}}\left(E_{1}\right) \otimes \cdots \otimes S^{\delta_{k}}\left(E_{k}\right)\right)$.

The natural geometric objects that are associated to this kind of problems are the higher Secant varieties of the SegreVeronese varieties that we are going to define.

Definition 2.6. Let $X \subset \mathbb{P}^{N}$ be any projective variety and define $X_{s}^{0}:=\bigcup_{\overline{\mathbf{P}_{\mathbf{1}}}, \ldots, \overline{\mathbf{P}_{\mathbf{s}}} \in X}\left\langle\overline{\mathbf{P}_{\mathbf{1}}}, \ldots, \overline{\mathbf{P}_{\mathbf{s}}}\right\rangle$. The $s$-th secant variety $X_{s} \subset \mathbb{P}^{N}$ of $X$ is the Zariski closure of $X_{s}^{0}$.
Observe that the generic element of $X_{s}$ is a point $\overline{\mathbf{P}} \in \mathbb{P}^{N}$ that can be written as a linear combination of $s$ points of $X$, in fact a generic element of $X_{s}$ is an element of $X_{s}^{0}$. Therefore if $X$ is the Segre-Veronese variety, then the generic element of $\Xi_{s}\left(S^{\delta_{1}}\left(E_{1}\right) \otimes \cdots \otimes S^{\delta_{k}}\left(E_{k}\right)\right)$ is the projective class of a partially symmetric tensor $T \in S^{\delta_{1}}\left(E_{1}\right) \otimes \cdots \otimes$ $S^{\delta_{k}}\left(E_{k}\right)$ that can be written as a linear combination of $s$ linearly independent partially symmetric tensors of rank 1 . Unfortunately not all the elements of $\Xi_{s}\left(S^{\delta_{1}}\left(E_{1}\right) \otimes \cdots \otimes\right.$ $\left.S^{\delta_{k}}\left(E_{k}\right)\right)$ are of this form. In fact if $\bar{T} \in \Xi_{s}\left(S^{\delta_{1}}\left(E_{1}\right) \otimes \cdots \otimes\right.$ $\left.S^{\delta_{k}}\left(E_{k}\right)\right) \backslash \Xi_{s}^{0}\left(S^{\delta_{1}}\left(E_{1}\right) \otimes \cdots \otimes S^{\delta_{k}}\left(E_{k}\right)\right)$ then the rank of $T$ is strictly bigger than $s$.

Definition 2.7. The minimum integer s such that $\bar{T} \in$ $\mathbb{P}\left(S^{\delta_{1}}\left(E_{1}\right) \otimes \cdots \otimes S^{\delta_{k}}\left(E_{k}\right)\right)$ belongs to $\Xi_{s}\left(S^{\delta_{1}}\left(E_{1}\right) \otimes \cdots \otimes\right.$ $\left.S^{\delta_{k}}\left(E_{k}\right)\right)$ is called the border rank of $T$.

In order to find the border rank of a tensor $T \in S^{\delta_{1}}\left(E_{1}\right) \otimes$ $\cdots \otimes S^{\delta_{k}}\left(E_{k}\right)$ we should need a set of equations for $\Xi_{s}\left(S^{\delta_{1}}\left(E_{1}\right)\right.$ $\left.\otimes \cdots \otimes S^{\delta_{k}}\left(E_{k}\right)\right)$ for $s>1$. The knowledge of the generators of the ideals of secant varieties of homogeneous varieties is a very deep problem that is solved only for very particular cases (see eg. [37], [34], [32], [33], [7], [?]).

From a computational point of view, there is a very direct and well known way of getting the equations for the secant variety, which consists of introducing parameters or unknowns for the coefficients of $\mathbf{l}_{i, j}$ and $\gamma_{i}$ in (2), to expand the polynomial and identify its coefficients with the coefficients of $T$. Eliminating the coefficients of $\mathbf{l}_{i, j}$ and $\gamma_{i}$ yields the equations of the secant variety.

Unfortunately this procedure is far from being computationally practical, because we have to deal with high degree polynomials in many variables, with a lot of symmetries. This is why we need to introduce moment matrices and to use a different kind of elimination.

\subsection{Moment matrices}

In this section, we recall the algebraic tools and the properties we need to describe and analyze our algorithm. We refer e.g. to [5], [23], [36].

Let $n:=\sum_{i} n_{i}$, we have $R \simeq \mathbb{K}\left[x_{1}, \ldots, x_{n}\right]$. For any $\Lambda \in$ $R^{*}$, we define the bilinear form $Q_{\Lambda}$, such that $\forall a, b \in R$, $Q(a, b)=\Lambda(a b)$. The matrix of $Q_{\Lambda}$ in the monomial basis, of $R$ is $\mathbb{Q}_{\Lambda}=\left(\Lambda\left(\mathbf{x}^{\alpha+\beta}\right)\right)_{\alpha, \beta}$, where $\alpha, \beta \in \mathbb{N}^{n}$. Similarly, for any $\Lambda \in R^{*}$, we define the Hankel operator $H_{\Lambda}$ from $R$ to $R^{*}$ as

$$
\begin{aligned}
H_{\Lambda}: \quad & R \rightarrow R^{*} \\
& p \mapsto p \star \Lambda .
\end{aligned}
$$

The matrix of the linear operator $H_{\Lambda}$ in the monomial basis, and in the dual basis, $\left\{\mathbf{d}^{\alpha}\right\}$, is $\mathbb{H}_{\Lambda}=\left(\Lambda\left(\mathbf{x}^{\alpha+\beta}\right)\right)_{\alpha, \beta}$, where $\alpha, \beta \in \mathbb{N}^{n}$. The following relates the Hankel operators with the bilinear forms. For all $a, b \in R$, thanks to the $R$-module structure, it holds

$$
Q_{\Lambda}(a, b)=\Lambda(a b)=a \star \Lambda(b)=H_{\Lambda}(a)(b) .
$$

In what follows, we will identify $H_{\Lambda}$ and $Q_{\Lambda}$.

Definition 2.8. Given $B=\left\{b_{1}, \ldots, b_{r}\right\}, B^{\prime}=\left\{b_{1}^{\prime}, \ldots\right.$, $\left.b_{r^{\prime}}^{\prime}\right\} \subset R$, we define

$$
H_{\Lambda}^{B, B^{\prime}}:\langle B\rangle \rightarrow\left\langle B^{\prime}\right\rangle^{*},
$$

as the restriction of $H_{\Lambda}$ to the vector space $\langle B\rangle$ and inclusion of $\left\langle B^{\prime}\right\rangle^{*}$ in $R^{*}$. Let $\mathbb{H}_{\Lambda}^{B, B^{\prime}}=\left(\Lambda\left(b_{i} b_{j}^{\prime}\right)\right)_{1 \leq i \leq r, 1 \leq j \leq r^{\prime}}$. If $B^{\prime}=$ $B$, we also use the notation $H_{\Lambda}^{B}$ and $\mathbb{H}_{\Lambda}^{\bar{B}}$. 
If $B, B^{\prime}$ are linearly independent, then $\mathbb{H}_{\Lambda}^{B, B^{\prime}}$ is the matrix of $H_{\Lambda}^{B, B^{\prime}}$ in this basis $\left\{b_{1}, \ldots, b_{r}\right\}$ of $\langle B\rangle$ and the dual basis of $B^{\prime}$ in $\left\langle B^{\prime}\right\rangle^{*}$. The catalecticant matrices of [27] correspond to the case where $k=1$ and $B$ and $B^{\prime}$ are, respectively, the set of monomials of degree $\leq i$ and $\leq d-i(i=0, \ldots, \delta)$.

From the definition of the Hankel operators, we can deduce that a polynomial $p \in R$ belongs to the kernel of $\mathbb{H}_{\Lambda}$ if and only if $p \star \Lambda=0$, which in turn holds if and only if for all $q \in R, \Lambda(p q)=0$.

Proposition 2.9 ([5]). Let $I_{\Lambda}$ be the kernel of $H_{\Lambda}$. Then, $I_{\Lambda}$ is an ideal of $R$.

Let $\mathcal{A}_{\Lambda}=R / I_{\Lambda}$ be the quotient algebra of polynomials modulo the ideal $I_{\Lambda}$, which, as Proposition 2.9 states is the kernel of $H_{\Lambda}$. The rank of $H_{\Lambda}$ is the dimension of $\mathcal{A}_{\Lambda}$ as a $\mathbb{K}$-vector space.

Definition 2.10. For any $B \subset R$, let $B^{+}=B \cup x_{1,1} B \cup$ $\cdots \cup x_{n_{k}, k} B$ and $\partial B=B^{+} \backslash B$.

Proposition $2.11 \quad([35,5])$. Assume that $\operatorname{rank}\left(H_{\Lambda}\right)=$ $r<\infty$ and let $B=\left\{b_{1}, \ldots, b_{r}\right\} \subset R$ such that $\mathbb{H}_{\Lambda}^{B}$ is invertible. Then $b_{1}, \ldots, b_{r}$ is a basis of $\mathcal{A}_{\Lambda}$. If $1 \in\langle B\rangle$ the ideal $I_{\Lambda}$ is generated by $\operatorname{ker} H_{\Lambda}^{B^{+}}$.

Proposition 2.12 ([23, 5]). If $\operatorname{rank}\left(H_{\Lambda}\right)=r<\infty$, then $\mathcal{A}_{\Lambda}$ is of dimension $r$ over $\mathbb{K}$ and there exist $\zeta_{1}, \ldots, \zeta_{d} \in$ $\mathbb{K}^{n}$ where $\left.d \leq r\right)$, and $p_{i} \in \mathbb{K}\left[\partial_{1}, \ldots, \partial_{n}\right]$, such that

$$
\Lambda=\sum_{i=1}^{d} \mathbf{1}_{\zeta_{i}} \circ p_{i}(\partial) .
$$

Moreover the multiplicity of $\zeta_{i}$ is the dimension of the vector space spanned the inverse system generated by $\mathbf{1}_{\zeta_{i}} \circ p_{i}(\partial)$.

In characteristic 0 , the inverse system of $\mathbf{1}_{\zeta_{i}} \circ p_{i}(\partial)$ is isomorphic to the vector space generated by $p_{i}$ and its derivatives of any order with respect to the variables $\partial_{i}$. In general characteristic, we replace the derivatives by the product by the "inverse" of the variables [36], [23].

Definition 2.13. For $T^{*} \in R_{\delta_{1}, \ldots, \delta_{k}}^{*}$, we call generalized decomposition of $T^{*}$ a decomposition such that $T^{*}=$ $\sum_{i=1}^{d} \mathbf{1}_{\zeta_{i}} \circ p_{i}(\partial)$ where the sum for $i=1, \ldots, d$ of the dimensions of the vector spaces spanned by the inverse system generated by $\mathbf{1}_{\zeta_{i}} \circ p_{i}(\partial)$ is minimal. This minimal sum of dimensions is called the length of $T^{*}$.

This definition extends the definition introduced in [27] for binary forms. The length of $T^{*}$ is the rank of the corresponding Hankel operator $H_{\Lambda}$.

Theorem 2.14 ([5]). For any $\Lambda \in R^{*}$, we have $\Lambda=$ $\sum_{i=1}^{r} \gamma_{i} \mathbf{1}_{\zeta_{i}}$ with $\gamma_{i} \neq 0$ and $\zeta_{i}$ distinct points of $\mathbb{K}^{n}$ iff rank $H_{\Lambda}=r$ and $I_{\Lambda}$ is a radical ideal.

In the binary case this rank also corresponds to the border rank of $T^{*}$, therefore the $r$-th minors of the Hankel operator give equations for the $r$-th secant variety to the rational normal curves [27].

In order to compute the zeroes of an ideal $I_{\Lambda}$ when we know a basis of $\mathcal{A}_{\Lambda}$, we exploit the properties of the operators of multiplication in $\mathcal{A}_{\Lambda}: M_{a}: \mathcal{A}_{\Lambda} \rightarrow \mathcal{A}_{\Lambda}$, such that $\forall b \in \mathcal{A}_{\Lambda}, M_{a}(b)=a b$ and its transposed operator $M_{a}^{t}: \mathcal{A}_{\Lambda}^{*} \rightarrow \mathcal{A}_{\Lambda}^{*}$, such that for $\forall \gamma \in \mathcal{A}_{\Lambda}^{*}, M_{a}^{\top}(\gamma)=a \star \gamma$.

The following proposition expresses a similar result, based on the properties of the duality.
Proposition $2.15 \quad([36,5])$. For any linear form $\Lambda \in$ $R^{*}$ such that rank $H_{\Lambda}<\infty$ and any $a \in \mathcal{A}_{\Lambda}$, we have

$$
H_{a \star \Lambda}=M_{a}^{t} \circ H_{\Lambda}
$$

We have the following well-known theorem:

Theorem $2.16 \quad([19,18,23])$. Assume that $\mathcal{A}_{\Lambda}$ is a finite dimensional vector space. Then $\Lambda=\sum_{i=1}^{d} \mathbf{1}_{\zeta_{i}} \circ p_{i}(\partial)$ for $\zeta_{i} \in \mathbb{K}^{n}$ and $p_{i}(\partial) \in \mathbb{K}\left[\partial_{1}, \ldots, \partial_{n}\right]$ and

- the eigenvalues of the operators $M_{a}$ and $M_{a}^{t}$, are given by $\left\{a\left(\zeta_{1}\right), \ldots, a\left(\zeta_{r}\right)\right\}$.

- the common eigenvectors of the operators $\left(M_{x_{i}}^{t}\right)_{1 \leq i \leq n}$ are (up to scalar) $\mathbf{1}_{\zeta_{i}}$.

Using the previous proposition, one can recover the points $\zeta_{i} \in \mathbb{K}^{n}$ by eigenvector computation as follows. Assume that $B \subset R$ with $|B|=\operatorname{rank}\left(H_{\Lambda}\right)$, then equation (6) and its transposition yield

$$
\mathbb{H}_{a \star \Lambda}^{B}=\mathbb{M}_{a}^{t} \mathbb{H}_{\Lambda}^{B}=\mathbb{H}_{\Lambda}^{B} \mathbb{M}_{a},
$$

where $\mathbb{M}_{a}$ is the matrix of multiplication by $a$ in the basis $B$ of $\mathcal{A}_{\Lambda}$. By Theorem 2.16, the common solutions of the generalized eigenvalue problem

$$
\left(\mathbb{H}_{a \star \Lambda}-\lambda \mathbb{H}_{\Lambda}\right) \mathbf{v}=\mathbb{O}
$$

for all $a \in R$, yield the common eigenvectors $\mathbb{H}_{\Lambda}^{B} \mathbf{v}$ of $\mathbb{M}_{a}^{t}$, that is the evaluation $\mathbf{1}_{\zeta_{i}}$ at the roots. Therefore, these common eigenvectors $\mathbb{H}_{\Lambda}^{B} \mathbf{v}$ are up to a scalar, the vectors $\left[b_{1}\left(\zeta_{i}\right), \ldots, b_{r}\left(\zeta_{i}\right)\right](i=1, \ldots, r)$. Notice that it is sufficient to compute the common eigenvectors of $\left(\mathbb{H}_{x_{i} \star \Lambda}, \mathbb{H}_{\Lambda}\right)$ for $i=$ $1, \ldots, n$

If $\Lambda=\sum_{i=1}^{d} \gamma_{i} \mathbf{1}_{\zeta_{i}}\left(\gamma_{i} \neq 0\right)$, then the roots are simple, and one eigenvector computation is enough: for any $a \in R, \mathbb{M}_{a}$ is diagonalizable and the generalized eigenvectors $\mathbb{H}_{\Lambda}^{B} \mathbf{v}$ are, up to a scalar, the evaluation $\mathbf{1}_{\zeta_{i}}$ at the roots.

Coming back to our problem of partially symmetric tensor decomposition, $T^{*} \in R_{\delta_{1}, \ldots, \delta_{k}}^{*}$ admits an affine decomposition of rank $r$ iff $T^{*}$ coincide on $R_{\delta_{1}, \ldots, \delta_{k}}$ with

$$
\Lambda=\sum_{i=1}^{r} \gamma_{i} \mathbf{1}_{\zeta_{i}},
$$

for some distinct $\zeta_{1}, \ldots, \zeta_{r} \in \mathbb{K}^{n_{1}} \times \cdots \times \mathbb{K}^{n_{k}}$ and some $\gamma_{i} \in \mathbb{K}-\{0\}$. Then, by theorem 2.14, $H_{\Lambda}$ is of rank $r$ and $I_{\Lambda}$ is radical.

Conversely, given $H_{\Lambda}$ of rank $r$ with $I_{\Lambda}$ radical which coincides on $R_{\delta_{1}, \ldots, \delta_{k}}$ with $T^{*}$, by proposition $2.12, \Lambda=$ $\sum_{i=1}^{r} \gamma_{i} \mathbf{1}_{\zeta_{i}}$ and extends $T^{*}$, which thus admits an affine decomposition.

Therefore we can say that if the border rank of $T$ is $r$ then also $\operatorname{rank}\left(H_{\Lambda}\right)=r$. Conversely if $\operatorname{rank}\left(H_{\Lambda}\right)=r$, we can only claim that the border rank of $T$ is at least $r$.

The problem of decomposition of $T^{*}$ can thus be reformulated as follows:

Truncated moment problem. Given $T^{*} \in R_{\delta_{1}, \ldots, \delta_{k}}^{*}$, find the smallest $r$ such that there exists $\Lambda \in R^{*}$ which extends $T^{*}$ with $H_{\Lambda}$ of rank $r$ and $I_{\Lambda}$ a radical ideal.

In the next section, we will describe an algorithm to solve the truncated moment problem.

\section{ALGORITHM}

In this section, we first describe the algorithm from a geometric point of view and the algebraic computation it induces. Then, we characterize under which the conditions $T^{*}$ 
can be extended to $\Lambda \in R^{*}$ with $H_{\Lambda}$ of rank $r$. The idea of the algorithm is the following:

Given a tensor $T \in S^{\delta_{1}}\left(E_{1}\right) \otimes \cdots \otimes S^{\delta_{k}}\left(E_{k}\right)$, set $r=0$;

1. Determine if $T^{*}$ can be extended to $\Lambda \in R^{*}$ with rank $H_{\Lambda}=r$; if the answer is YES, go to step 2, otherwise repeat step 1) with $r+1$.

2. Find if there exists $r$ distinct points $P_{1}, \ldots, P_{r} \in$ $\Xi\left(S^{\delta_{1}}\left(E_{1}\right) \otimes \cdots \otimes S^{\delta_{k}}\left(E_{k}\right)\right)$ such that $T \in\left\langle P_{1}, \ldots, P_{r}\right\rangle \simeq$ $\mathbb{P}^{s-1}$ - equivalently compute the roots of ker $H_{\Lambda}$ by generalized eigenvector computation (7) and check that the eigenspaces are simple;

3. If the answer to 2 is YES, then the rank of $T$ is actually $r$ and we are done;

4. If the answer to 3 is NO, then it means that the rank of $T$ is bigger than $r$. Repeat this procedure from step 3 with $r+1$.

This algorithm extends the one in [5] which applies only for symmetric tensors. The approach used in [3] for the rank of tensors in $\Xi_{2}\left(S^{d}(E)\right)$ and in $\Xi_{3}\left(S^{d}(E)\right)$ allows to avoid to loop again at step 4: if one doesn't get simple roots, then it is possible to use other techniques to compute the rank. Unfortunately the mathematical knowledge on the stratification by rank of secant varieties is nowadays not complete, hence these techniques cannot be used now to improve algorithms for higher border ranks.

We are going to characterize now under which conditions $T^{*}$ can be extended to $\Lambda \in R^{*}$ with $H_{\Lambda}$ of rank $r$ (step 3).

We need the following technical property on the bases of $\mathcal{A}_{\Lambda}$, that we will consider:

DeFinition 3.1. Let $B$ be a subset of monomials in $R \simeq$ $\mathbb{K}\left[x_{1}, \ldots, x_{n}\right]$. We say that $B$ is connected to 1 if $\forall m \in B$ either $m=1$ or there exists $i \in[1, n]$ and $m^{\prime} \in B$ such that $m=x_{i} m^{\prime}$.

Let $B, B^{\prime} \subset R_{\delta_{1}, \ldots, \delta_{k}}$ be a two sets of monomials connected to 1 . We consider the formal Hankel matrix

$$
\mathcal{H}_{\Lambda}^{B, B^{\prime}}=\left(h_{\alpha+\beta}\right)_{\alpha \in B^{\prime}, \beta \in B},
$$

with $h_{\alpha}=T^{*}\left(\mathbf{x}^{\alpha}\right)=c_{\alpha}$ if $\mathbf{x}^{\alpha} \in R_{\delta_{1}, \ldots, \delta_{k}}$ and otherwise $h_{\alpha}$ is a variable. The set of these new variables is denoted $\mathbf{h}$.

Suppose that $\mathcal{H}_{\Lambda}^{B, B^{\prime}}$ is invertible in $\mathbb{K}(\mathbf{h})$, then we define the formal multiplication operators

$$
\mathcal{M}_{i, l}^{B, B^{\prime}}(\mathbf{h}):=\left(\mathcal{H}_{\Lambda}^{B, B^{\prime}}\right)^{-1} \mathcal{H}_{x_{i, l} \star B^{\prime} \Lambda}
$$

for every variable $x_{i, l} \in R$.

We use the following theorems which extend the results of [35] to the cases of distinct sets of monomials indexing the rows and columns of the Hankel operators. They characterizes the cases where $\mathbb{K}[\mathbf{x}]=B \oplus I_{\Lambda}$ :

Theorem 3.2. Let $B=\left\{\mathbf{x}^{\beta_{1}}, \ldots, \mathbf{x}^{\beta_{r}}\right\}$ and $B^{\prime}=\left\{\mathbf{x}^{\beta_{1}^{\prime}}\right.$, $\left.\ldots, \mathbf{x}^{\beta_{r}^{\prime}}\right\}$ be two sets of monomials in $R_{\delta_{1}, \ldots, \delta_{k}}$, connected to 1 and let $\Lambda$ be a linear form that belongs to $\left(\left\langle B^{\prime} \cdot B^{+}\right\rangle_{\delta_{1}, \ldots, \delta_{k}}\right)^{*}$. Let $\Lambda(\mathbf{h})$ be the linear form of $\left\langle B^{\prime} \cdot B^{+}\right\rangle^{*}$ defined by $\Lambda(\mathbf{h})\left(\mathbf{x}^{\alpha}\right)=$ $\Lambda\left(\mathbf{x}^{\alpha}\right)$ if $\mathbf{x}^{\alpha} \in R_{\delta_{1}, \ldots, \delta_{k}}$ and $h_{\alpha} \in \mathbb{K}$ otherwise. Then, $\Lambda(\mathbf{h})$ admits an extension $\tilde{\Lambda} \in R^{*}$ such that $H_{\tilde{\Lambda}}$ is of rank $r$ with $B$ and $B^{\prime}$ basis of $A_{\tilde{\Lambda}}$ iff

$$
\mathcal{M}_{i, l}^{B}(\mathbf{h}) \circ \mathcal{M}_{j, q}^{B}(\mathbf{h})-\mathcal{M}_{j, q}^{B}(\mathbf{h}) \circ \mathcal{M}_{i, l}^{B}(\mathbf{h})=0
$$

$\left(0 \leq l, q \leq k, 1 \leq i \leq n_{l}, 1 \leq j \leq n_{q}\right)$ and $\operatorname{det}\left(\mathcal{H}_{\Lambda(\mathbf{h})}^{B^{\prime}, B}\right) \neq 0$. Moreover, such a $\tilde{\Lambda}$ is unique.
We are going to give an equivalent characterization of the extension property, based on rank conditions:

Theorem 3.3. Let $B=\left\{\mathbf{x}^{\beta_{1}}, \ldots, \mathbf{x}^{\beta_{r}}\right\}$ and $B^{\prime}=\left\{\mathbf{x}^{\beta_{1}^{\prime}}\right.$, $\left.\ldots, \mathbf{x}^{\beta_{r}^{\prime}}\right\}$ be two sets of monomials in $R_{\delta_{1}, \ldots, \delta_{k}}$, connected to 1. Let $\Lambda$ be a linear form in $\left(\left\langle B^{\prime+} * B^{+}\right\rangle_{\delta_{1}, \ldots, \delta_{k}}\right)^{*}$ and $\Lambda(\mathbf{h})$ be the linear form of $\left\langle B^{\prime+} \cdot B^{+}\right\rangle^{*}$ defined by $\Lambda(\mathbf{h})\left(\mathbf{x}^{\alpha}\right)=\Lambda\left(\mathbf{x}^{\alpha}\right)$ if $\mathbf{x}^{\alpha} \in R_{\delta_{1}, \ldots, \delta_{k}}$ and $h_{\alpha} \in \mathbb{K}$ otherwise. Then, $\Lambda(\mathbf{h})$ admits an extension $\tilde{\Lambda} \in R^{*}$ such that $H_{\tilde{\Lambda}}$ is of rank $r$ with $B$ and $B^{\prime}$ basis of $A_{\tilde{\Lambda}}$ iff all $(r+1) \times(r+1)$-minors of $\mathcal{H}_{\Lambda(\mathbf{h})}^{B^{\prime+}, B^{+}}$ vanish and $\operatorname{det}\left(\mathcal{H}_{\Lambda(\mathbf{h})}^{B^{\prime}, B}\right) \neq 0$.

\section{EXAMPLES AND APPLICATIONS}

There exist numerous fields in which decomposing a tensor into a sum of rank-one terms is useful. These fields range from arithmetic complexity [8] to chemistry [42]. One nice application is worth to be emphasized, namely wireless transmissions [40]: one or several signals are wished to be extracted form noisy measurements, received on an array of sensors and disturbed by interferences. The approach is deterministic, which makes the difference compared to approaches based on data statistics [15]. The array of sensors is composed of $J$ subarrays, each containing $I$ sensors. Subarrays do not need to be disjoint, but must be deduced from each other by a translation in space. If the transmission is narrow band and in the far field, then the measurements at time sample $t$ recorded on sensor $i$ of subarray $j$ take the form:

$$
T(i, j, t)=\sum_{p=1}^{r} A_{i p} B_{j p} C_{t p}
$$

if $r$ waves impinge on the array. Matrices $A$ and $B$ characterize the geometry of the array (subarray and translations), whereas matrix $C$ contains the signals received on the array. An example with $(I, J)=(4,4)$ is given in Figure 1. Computing the decomposition of tensor $T$ allows to extract signals of interest as well as interferences, all included in matrix $C$. Radiating sources can also be localized with the help of matrix $A$ if the exact location of sensors of a subarray are known. Note that this framework applies in radar, sonar or telecommunications.

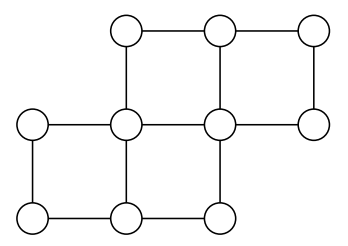

Figure 1: Array of 10 sensors decomposed into 4 subarrays of 4 sensors each.

We consider such an example with 6 time samples, that is an element of $\mathbb{R}^{4} \otimes \mathbb{R}^{4} \otimes \mathbb{R}^{6}: T:=1046 a_{1} b_{1} c_{1}+959 a_{1} b_{1} c_{2}+$ $660 a_{1} b_{1} c_{3}+866 a_{1} b_{1} c_{4}+952 a_{1} b_{1} c_{5}-1318 a_{1} b_{2} c_{1}-1222 a_{1} b_{2} c_{2}-906 a_{1} b_{2} c_{3}-$ $1165 a_{1} b_{2} c_{4}-1184 a_{1} b_{2} c_{5}-153 a_{1} b_{3} c_{1}+52 a_{1} b_{3} c_{2}+353 a_{1} b_{3} c_{3}+354 a_{1} b_{3} c_{4}+$ $585 a_{1} b_{3} c_{5}+852 a_{2} b_{1} c_{1}+833 a_{2} b_{1} c_{2}+718 a_{2} b_{1} c_{3}+903 a_{2} b_{1} c_{4}+828 a_{2} b_{1} c_{5}-$ $1068 a_{2} b_{2} c_{1}-1060 a_{2} b_{2} c_{2}-992 a_{2} b_{2} c_{3}-1224 a_{2} b_{2} c_{4}-1026 a_{2} b_{2} c_{5}+$ $256 a_{2} b_{3} c_{1}+468 a_{2} b_{3} c_{2}+668 a_{2} b_{3} c_{3}+748 a_{2} b_{3} c_{4}+1198 a_{2} b_{3} c_{5}-614 a_{3} b_{1} c_{1}-$ $495 a_{3} b_{1} c_{2}-276 a_{3} b_{1} c_{3}-392 a_{3} b_{1} c_{4}-168 a_{3} b_{1} c_{5}+664 a_{3} b_{2} c_{1}+525 a_{3} b_{2} c_{2}+$ $336 a_{3} b_{2} c_{3}+472 a_{3} b_{2} c_{4}+63 a_{3} b_{2} c_{5}+713 a_{3} b_{3} c_{1}+737 a_{3} b_{3} c_{2}+791 a_{3} b_{3} c_{3}+$ $965 a_{3} b_{3} c_{4}+674 a_{3} b_{3} c_{5}-95 a_{1} b_{1}+88 a_{1} b_{2}+193 a_{1} b_{3}+320 a_{1} c_{1}+285 a_{1} c_{2}+$ $134 a_{1} c_{3}+188 a_{1} c_{4}+382 a_{1} c_{5}-29 a_{2} b_{1}-2 a_{2} b_{2}+198 a_{2} b_{3}+292 a_{2} c_{1}+$ $269 a_{2} c_{2}+138 a_{2} c_{3}+187 a_{2} c_{4}+406 a_{2} c_{5}+119 a_{3} b_{1}-139 a_{3} b_{2}+20 a_{3} b_{3}-$ 
$222 a_{3} c_{1}-160 a_{3} c_{2}+32 a_{3} c_{3}+9 a_{3} c_{4}-229 a_{3} c_{5}+122 b_{1} c_{1}+119 b_{1} c_{2}+$ $112 b_{1} c_{3}+140 b_{1} c_{4}+108 b_{1} c_{5}-160 b_{2} c_{1}-163 b_{2} c_{2}-176 b_{2} c_{3}-214 b_{2} c_{4}-$ $117 b_{2} c_{5}+31 b_{3} c_{1}+57 b_{3} c_{2}+65 b_{3} c_{3}+73 b_{3} c_{4}+196 b_{3} c_{5}-35 a_{1}-21 a_{2}+$ $54 a_{3}-3 b_{1}-3 b_{2}+24 b_{3}+50 c_{1}+46 c_{2}+20 c_{3}+29 c_{4}+63 c_{5}-6$

If we take $B=\left\{1, a_{1}, a_{2}, a_{3}, b_{1}, b_{2}\right\}$ and $B^{\prime}=\left\{1, c_{1}, c_{2}, c_{3}\right.$, $\left.c_{4}, c_{5}\right\}$ we obtain the following known submatrix of $H_{\Lambda}$ :

$$
\mathbb{H}_{\Lambda}^{B^{\prime}, B}=\left[\begin{array}{cccccc}
-6 & -35 & -21 & 54 & -3 & -3 \\
50 & 320 & 292 & -222 & 122 & -160 \\
46 & 285 & 269 & -160 & 119 & -163 \\
20 & 134 & 138 & 32 & 112 & -176 \\
29 & 188 & 187 & 9 & 140 & -214 \\
63 & 382 & 406 & -229 & 108 & -117
\end{array}\right]
$$

which is invertible. Thus, the rank is at least 6 . Let us find if $H_{\tilde{\Lambda}}$ can be extended to a rank 6 Hankel matrix $H_{\Lambda}$. If we look at $H_{\Lambda}^{B^{\prime+}, B^{+}}$, several coefficients are unknown. Yet, as will see, they can be determined by exploiting the commutation relations, as follows.

The columns $\mathbb{H}^{B^{\prime},\{m\}}$ are also known for $m \in\left\{b_{3}, a_{1} b_{1}\right.$, $\left.a_{2} b_{1}, a_{3} b_{1}, a_{1} b_{2}, a_{2} b_{2}, a_{3} b_{2}\right\}$. Thus we deduce the relations between these monomials and $B$ by solving the system

$\mathbb{H}_{\Lambda}^{B^{\prime}, B} X=\mathbb{H}_{\Lambda}^{B^{\prime},\{m\}}$. This yields the following relations in $\mathcal{A}_{\Lambda}: b_{3} \equiv-1 .-0.02486 a_{1}+1.412 a_{2}+0.8530 a_{3}-0.6116 b_{1}+0.3713 b_{2}, a_{1} b_{1} \equiv$ $-2 .+6.122 a_{1}-3.304 a_{2}+.6740 a_{3}+.7901 b_{1}-1.282 b_{2}, a_{2} b_{1} \equiv-2 .+4.298 a_{1}-$ $1.546 a_{2}+1.364 a_{3}+.5392 b_{1}-1.655 b_{2}, a_{3} b_{1} \equiv-2 .-3.337 a_{1}+5.143 a_{2}+$ $1.786 a_{3}-2.291 b_{1}+1.699 b_{2}, a_{1} b_{2} \equiv-2 .+0.03867 a_{1}-0.1967 a_{2}+1.451 a_{3}-$ $2.049 b_{1}+3.756 b_{2}, a_{2} b_{2} \equiv-2 .+3.652 a_{1}-3.230 a_{2}+.9425 a_{3}-2.562 b_{1}+$ $4.198 b_{2}, a_{3} b_{2} \equiv-2 .+6.243 a_{1}-7.808 a_{2}-1.452 a_{3}+5.980 b_{1}+0.03646 b_{2}$

Using the first relation on $b_{3}$, we can reduce $a_{1} b_{3}, a_{2} b_{3}, a_{3} b_{3}$ and obtain 3 linear dependency relations between the monomials in $B \cup\left\{a_{1}^{2}, a_{1} a_{2}, a_{1} a_{3}, a_{2}^{2}, a_{2} a_{3}, a_{3}^{2}\right\}$. Using the commutation relations $\frac{\operatorname{lcm}\left(m_{1}, m_{2}\right)}{m_{1}} N\left(m_{1}\right)-\frac{\operatorname{lcm}\left(m_{1}, m_{2}\right)}{m_{2}} N\left(m_{2}\right)$, for $\left(m_{1}, m_{2}\right) \in\left\{\left(a_{1} b_{1}, a_{2} b_{1}\right),\left(a_{1} b_{2}, a_{2} b_{2}\right),\left(a_{2} b_{2}, a_{3} b_{2}\right)\right\}$ where $N\left(m_{i}\right)$ is the reduction of $m_{i}$ with respect to the prevision relations, we obtain 3 new linear dependency relations between the monomials in $B \cup\left\{a_{1}^{2}, a_{1} a_{2}, a_{1} a_{3}, a_{2}^{2}, a_{2} a_{3}, a_{3}^{2}\right\}$. From these 6 relations, we deduce the expression of the monomials in $\left\{a_{1}^{2}, a_{1} a_{2}, a_{1} a_{3}, a_{2}^{2}, a_{2} a_{3}, a_{3}^{2}\right\}$ as linear combinations of monomials in $B$ :

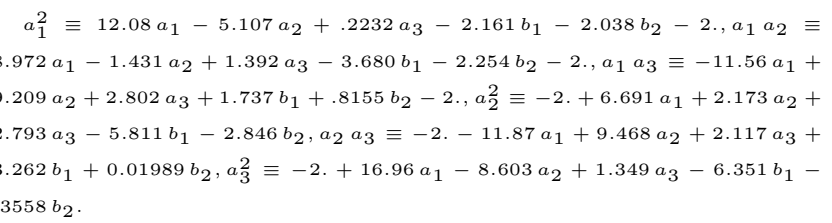

Now, we are able to compute the matrix of multiplication by $a_{1}$ in $B$, which is obtained by reducing the monomials $B \cdot a_{1}=\left\{a_{1}, a_{1}^{2}, a_{1} a_{2}, a_{1} a_{3}, a_{1} b_{1}, a_{1} b_{2}\right\}$ by the computed relations:

$$
M_{a_{1}}:=\left[\begin{array}{cccccc}
0.0 & -2.0 & -2.0 & -2.0 & -2.0 & -2.0 \\
1.0 & 12.08 & 8.972 & -11.56 & 6.122 & 0.03867 \\
0.0 & -5.107 & -1.431 & 9.209 & -3.304 & -0.1967 \\
0.0 & 0.2232 & 1.392 & 2.802 & 0.6740 & 1.451 \\
0.0 & -2.161 & -3.680 & 1.737 & 0.7901 & -2.049 \\
0.0 & -2.038 & -2.254 & 0.8155 & -1.282 & 3.756
\end{array}\right]
$$

The eigenvectors of the transposed operator are:

$$
\left[\begin{array}{c}
1.0 \\
5.0 \\
7.003 \\
3.0 \\
3.0 \\
-4.0
\end{array}\right],\left[\begin{array}{c}
1.0 \\
2.999 \\
4.0 \\
-4.999 \\
-2.999 \\
4.999
\end{array}\right],\left[\begin{array}{l}
1.0 \\
2.0 \\
2.0 \\
2.0 \\
2.0 \\
2.0
\end{array}\right],\left[\begin{array}{c}
1.0 \\
8.001 \\
6.002 \\
-7.002 \\
4.001 \\
-5.001
\end{array}\right],\left[\begin{array}{c}
1.0 \\
-1.0 \\
-2.0 \\
3.0 \\
-1.0 \\
-1.0
\end{array}\right],\left[\begin{array}{c}
1.0 \\
0.9999 \\
0.9999 \\
0.9999 \\
0.9999 \\
0.9999
\end{array}\right]
$$

They are normalized so that the first coordinate is 1 and correspond to the vectors of evaluation of the monomial vector $B$ at the roots of $I_{\Lambda}$. Thus we known the coordinates $a_{1}, a_{2}, a_{3}, b_{1}, b_{2}$ of these roots. By expanding the polynomial

$\left.\gamma_{1}\left(1+a_{1}+a_{2}+a_{3}\right)\right)\left(1+b_{1}+b_{2}+b_{3}\right)(1+\cdots)+\gamma_{2}\left(1-a_{1}-2 a_{2}+3 a_{3}\right)\left(1-b_{1}-\right.$ $\left.b_{2}-b_{3}\right)(1+\cdots)+\gamma_{3}\left(1+2 a_{1}+2 a_{2}+2 a_{3}\right)\left(1+2 b_{1}+2 b_{2}+3 b_{3}\right)(1+\cdots)+\gamma_{4}(1+$ $\left.5 a_{1}+7 a_{2}+3 a_{3}\right)\left(1+3 b_{1}-4 b_{2}+8 b_{3}\right)(1+\cdots)+\gamma_{5}\left(1+8 a_{1}+6 a_{2}-7 a_{3}\right)(1+$ $\left.4 b_{1}-5 b_{2}-3 b_{3}\right)(1+\cdots)+\gamma_{6}\left(1+3 a_{1}+4 a_{2}-5 a_{3}\right)\left(1-3 b_{1}+5 b_{2}+4 b_{3}\right)(1+\cdots)$ (where the $\cdots$ are terms linear in $c_{i}$ ) and identifying the coefficients of $T$ which do not depend on $c_{1}, \ldots, c_{5}$, we obtain a linear system in $\gamma_{i}$, which unique solution is $(2,-1,-2,3$, $-5,-3)$. This allows us to compute the value $\Lambda$ for any monomials in $\left\{a_{1}, a_{2}, a_{3}, b_{1}, b_{2}, b_{3}\right\}$. In particular, we can compute the entries of $\mathbb{H}_{\Lambda}^{B, B}$. Solving the system $\mathbb{H}_{\Lambda}^{B, B} X=$ $\mathbb{H}_{\Lambda}^{B, B^{\prime}}$, we deduce the relations between the monomials in $B^{\prime}$ and $B$ in $\mathcal{A}_{\Lambda}$ and in particular $c_{1}, \ldots, c_{5}$ as linear combinations of monomials in $B$. This allows us to recover the missing coordinates and yields the following decomposition: $T:=2\left(1+a_{1}+a_{2}+a_{3}\right)\left(1+b_{1}+b_{2}+b_{3}\right)\left(1+c_{1}+c_{2}+c_{3}+c_{4}+c_{5}\right)-(1-$ $\left.a_{1}-2 a_{2}+3 a_{3}\right)\left(1-b_{1}-b_{2}-b_{3}\right)\left(1-c_{1}-2 c_{2}-3 c_{3}-4 c_{4}+5 c_{5}\right)-2\left(1+2 a_{1}+\right.$ $\left.2 a_{2}+2 a_{3}\right)\left(1+2 b_{1}+2 b_{2}+3 b_{3}\right)\left(1+2 c_{1}+2 c_{2}+2 c_{3}+2 c_{4}+2 c_{5}\right)+3\left(1+5 a_{1}+\right.$ $\left.7 a_{2}+3 a_{3}\right)\left(1+3 b_{1}-4 b_{2}+8 b_{3}\right)\left(1+4 c_{1}+5 c_{2}+6 c_{3}+7 c_{4}+8 c_{5}\right)-5(1+$ $\left.8 a_{1}+6 a_{2}-7 a_{3}\right)\left(1+4 b_{1}-5 b_{2}-3 b_{3}\right)\left(1-6 c_{1}-5 c_{2}-2 c_{3}-3 c_{4}-5 c_{5}\right)-$ $3\left(1+3 a_{1}+4 a_{2}-5 a_{3}\right)\left(1-3 b_{1}+5 b_{2}+4 b_{3}\right)\left(1-3 c_{1}-2 c_{2}+3 c_{3}+3 c_{4}-7 c_{5}\right)$.

\section{REFERENCES}

[1] E. Ballico and A. Bernardi. Stratification of the fourth secant variety of veronese variety via symmetric rank. arXiv 1005.3465, 2010.

[2] A. Bernardi. Ideals of varieties parameterized by certain symmetric tensors. J. Pure Appl. Algebra, 212(6):1542-1559, 2008.

[3] A. Bernardi, A. Gimigliano, and M. Idà. Computing symmetric rank for symmetric tensors. J. Symb. Comput., 46:34-53, January 2011.

[4] D. Bini, M. Capovani, F. Romani, and G. Lotti. $O\left(n^{2.77}\right)$ Complexity for $n \times n$ approximate matrix multiplication. Inform. Process, 8(5):234-235, 1979.

[5] J. Brachat, P. Comon, B. Mourrain, and E. Tsigaridas. Symmetric tensor decomposition. Linear Algebra and Applications, 433:1851-1872, 2010.

[6] R. Bro. Parafac, tutorial and applications. Chemom. Intel. Lab. Syst., 38:149-171, 1997.

[7] J. Buczynski, A. Ginensky, and J.M. Landsberg. Determinental equations for secant varieties and the Eisenbud-Koh-Stillman conjecture. 1007.0192, 2010.

[8] P. Bürgisser, M. Clausen, and M. A. Shokrollahi. Algebraic complexity theory, volume 315 of Grundlehren der Mathematischen Wissenschaften [Fundamental Principles of Mathematical Sciences]. Springer-Verlag, Berlin, 1997. With the collaboration of Thomas Lickteig.

[9] J. F. Cardoso. Blind signal separation: statistical principles. Proc. of the IEEE, 90:2009-2025, October 1998. special issue, R.W. Liu and L. Tong eds.

[10] P. Chevalier. Optimal separation of independent narrow-band sources - concept and performance. Signal Processing, Elsevier, 73(1):27-48, February 1999. special issue on blind separation and deconvolution.

[11] P. Chevalier, L. Albera, A. Ferreol, and P. Comon. On the virtual array concept for higher order array 
processing. IEEE Proc., 53(4):1254-1271, April 2005.

[12] A. Cichocki and S-I. Amari. Adaptive Blind Signal and Image Processing. Wiley, New York, 2002.

[13] G. Comas and M. Seiguer. On the rank of a binary form, 2001.

[14] P. Comon. Independent Component Analysis. In J-L. Lacoume, editor, Higher Order Statistics, pages 29-38. Elsevier, Amsterdam, London, 1992.

[15] P. Comon and C. Jutten, editors. Handbook of Blind Source Separation, Independent Component Analysis and Applications. Academic Press, Oxford UK, Burlington USA, 2010.

[16] P. Comon and B. Mourrain. Decomposition of quantics in sums of powers of linear forms. Signal Processing, 53(2-3):93-107, 1996.

[17] P. Comon and M. Rajih. Blind identification of under-determined mixtures based on the characteristic function. Signal Processing, 86(9):2271-2281, 2006.

[18] D. Cox, J. Little, and D. O'Shea. Ideals, Varieties, and Algorithms. Undergraduate Texts in Mathematics. Springer-Verlag, New York, 2nd edition, 1997.

[19] D. Cox, J. Little, and D. O'Shea. Using Algebraic Geometry. Number 185 in Graduate Texts in Mathematics. Springer, New York, 2nd edition, 2005.

[20] L. de Lathauwer and J. Castaing. Tensor-based techniques for the blind separation of ds-cdma signals. Signal Processing, 87(2):322-336, February 2007.

[21] M. C. Dogan and J. Mendel. Applications of cumulants to array processing .I. aperture extension and array calibration. IEEE Trans. Sig. Proc., 43(5):1200-1216, May 1995.

[22] D. L. Donoho and X. Huo. Uncertainty principles and ideal atomic decompositions. IEEE Trans. Inform. Theory, 47(7):2845-2862, November 2001.

[23] M. Elkadi and B. Mourrain. Introduction à la résolution des systèmes polynomiaux, volume 59 of Mathmiatiques et Applications. Springer, 2007.

[24] A. Ferreol and P. Chevalier. On the behavior of current second and higher order blind source separation methods for cyclostationary sources. IEEE Trans. Sig. Proc., 48:1712-1725, June 2000. erratum in vol.50, pp.990, Apr. 2002.

[25] A. V. Geramita. Catalecticant varieties. In Commutative algebra and algebraic geometry (Ferrara), volume 206 of Lecture Notes in Pure and Appl. Math., pages 143-156. Dekker, New York, 1999.

[26] R. Grone. Decomposable tensors as a quadratic variety. Proc. Amer. Math. Soc., 64(2):227-230, 1977.

[27] H. T. Hà. Box-shaped matrices and the defining ideal of certain blowup surfaces. J. Pure Appl. Algebra, 167(2-3):203-224, 2002.

[28] A. Iarrobino and V. Kanev. Power sums, Gorenstein algebras, and determinantal loci, volume 1721 of Lecture Notes in Computer Science. Springer-Verlag, Berlin, 1999.

[29] T. Jiang and N. Sidiropoulos. Kruskal's permutation lemma and the identification of CANDECOMP/PARAFAC and bilinear models. IEEE Trans. Sig. Proc., 52(9):2625-2636, September 2004.

[30] H. A. L. Kiers and W. P. Krijnen. An efficient algorithm for Parafac of three-way data with large numbers of observation units. Psychometrika, 56:147, 1991.

[31] J. B. Kruskal. Three-way arrays: Rank and uniqueness of trilinear decompositions. Linear Algebra and Applications, 18:95-138, 1977.

[32] J. Landsberg. Geometry and the complexity of matrix multiplication. Bull. Amer. Math. Soc., 45(2):247-284, April 2008.

[33] J. M. Landsberg and L. Manivel. Generalizations of Strassen's equations for secant varieties of Segre varieties. Comm. Algebra, 36(2):405-422, 2008.

[34] J. M. Landsberg and G. Ottaviani. Equations for secant varieties to veronese varieties. arXiv 1006.0180, 2010.

[35] J. M. Landsberg and G. Ottaviani. Equations for secant varieties via vector bundles. arXiv 1010.1825, 2010.

[36] J. M. Landsberg and J. Weyman. On the ideals and singularities of secant varieties of Segre varieties. Bull. Lond. Math. Soc., 39(4):685-697, 2007.

[37] M. Laurent and B. Mourrain. A Sparse Flat Extension Theorem for Moment Matrices. Archiv der Mathematik, 93:87-98, 2009.

[38] B. Mourrain and V.Y. Pan. Multivariate Polynomials, Duality, and Structured Matrices. Journal of Complexity, 16(1):110-180, 2000.

[39] G. Ottaviani. An invariant regarding Waring's problem for cubic polynomials. Nagoya Math. J., 193:95-110, 2009.

[40] A. Parolin. Varietà secanti alle varietà di segre e di veronese e loro applicazioni, tesi di dottorato. Università di Bologna, 2003/2004.

[41] M. Pucci. The Veronese variety and catalecticant matrices. J. Algebra, 202(1):72-95, 1998.

[42] N. D. Sidiropoulos, R. Bro, and G. B. Giannakis. Parallel factor analysis in sensor array processing. IEEE Trans. Sig. Proc., 48(8):2377-2388, August 2000.

[43] N. D. Sidiropoulos, G. B. Giannakis, and R. Bro. Blind PARAFAC receivers for DS-CDMA systems. IEEE Trans. on Sig. Proc., 48(3):810-823, 2000.

[44] A. Smilde, R. Bro, and P. Geladi. Multi-Way Analysis. Wiley, 2004.

[45] V. Strassen. Rank and optimal computation of generic tensors. Linear Algebra Appl., 52:645-685, July 1983.

[46] A. Swami, G. Giannakis, and S. Shamsunder. Multichannel ARMA processes. IEEE Trans. Sig. Proc., 42(4):898-913, April 1994.

[47] J. J. Sylvester. Sur une extension d'un théorème de Clebsch relatif aux courbes du quatrième degré. Comptes Rendus, Math. Acad. Sci. Paris, 102:1532-1534, 1886.

[48] A. J. van der Veen and A. Paulraj. An analytical constant modulus algorithm. IEEE Trans. Sig. Proc., 44(5):1136-1155, May 1996.

[49] K. Wakeford. On canonical forms. Proc. London Math. Soc., 18:403-410, 1918-19. 